\title{
Resolution acute respiratory distress syndrome through reversing the imbalance of Treg/Th17 by targeting the cAMP signaling pathway
}

\author{
QIAN LI, XIUPING HU, RENHUA SUN, YUEXING TU, FANGXIAO GONG and YIN NI
}

\author{
Department of Critical Care Medicine, Zhejiang Provincial People's Hospital, Hangzhou, Zhejiang 310014, P.R. China
}

Received May 27, 2015; Accepted April 11, 2016

DOI: $10.3892 / \mathrm{mmr} .2016 .5222$

\begin{abstract}
Acute respiratory distress syndrome (ARDS) is a severe cause of respiratory failure with a mortality rate as high as $40-46 \%$ and without any effective pharmacological treatment available. The present study provided a novel strategy for the treatment of ARDS by specifically interfering with cyclic adenosine monophosphate (cAMP) signaling. Pre-treatment with the phosphodiesterase antagonist pentoxifyllinum (PTX) obviously attenuated lung injury and reduced the mortality of mice with cecal ligature and puncture (CLP)-induced ARDS, while raising cAMP levels. In addition, pre-treatment with PTX attenuated CLP-induced increases in the number of T-regulatory cells (Tregs) and interleukin (IL)-17-producing T-helper lymphocytes (Th17) among spleen lymphocytes, while partially restoring the Treg/Th17 ratio. Correspondingly, CLP-induced increases in the secretion of IL-2, IL-6, IL-10 and IL-17 were attenuated. Furthermore, CLP-induced increases in forkhead box p3 and RAR-related orphan receptor $\gamma \mathrm{t}(\mathrm{ROR} \gamma \mathrm{t})$ expression as well as signal transducer and activator of transcription (STAT3) activation were attenuated by PTX. The results indicated that PTX-induced increases in cAMP may have partly restored the Treg/Th17 balance by modulating the transcription of Foxp3 and ROR $\gamma$ t through the STAT3 pathway. In conclusion, the present study provided a novel treatment strategy for ARDS by modulating the balance of Treg/Th17 and the subsequent immune response via cAMP signaling, which requires pre-clinical and clinical validation.
\end{abstract}

\section{Introduction}

Acute respiratory distress syndrome (ARDS) a syndrome of acute respiratory failure, which was first described in 1967 by Ashbaugh et al (1). Affected patients present with progressive

Correspondence to: Dr Qian Li, Department of Critical Care Medicine, Zhejiang Provincial People's Hospital, 158 Shangtang Road, Hangzhou, Zhejiang 310014, P.R. China

E-mail: zjicudr@126.com

Key words: acute respiratory distress syndrome, Treg, Th17, forkhead box P3, cyclic adenosine monophosphate arterial hypoxemia, dyspnea and a marked increase in the work of breathing (2), and endotracheal intubation and positive pressure ventilation are required for most patients. ARDS may arise from a variety of medical conditions, including sepsis, pneumonia, major trauma or aspiration of gastric contents. Inappropriate accumulation and activity of leukocytes and platelets, dysregulated inflammation, altered permeability of alveolar endothelial and epithelial barriers and uncontrolled activation of coagulation pathways constitute the central pathophysiologies of ARDS. In spite of substantial progress in understanding underlying pathophysiology of ARDS, it remains a major clinical problem with the mortality rate remaining as high as 40-46\% and with no effective pharmacological therapy available (3). However, cell-based therapy has emerged as a promising therapeutic approach for ARDS (4).

ARDS was recently revealed to be an immune disorder arising from an imbalance between regulatory $\mathrm{T}$ cells (Tregs) and interleukin (IL)-17-producing $\mathrm{T}$ helper lymphocytes (Th17) (3). Th17 cells are known to have crucial roles in the induction of autoimmune diseases, including rheumatoid arthritis, experimental autoimmune encephalomyelitis and allergen-specific responses. Furthermore, Th17 cells are crucial for defense against extracellular bacteria and fungi $(5,6)$. Tregs are characterized by the forkhead box P3 (Foxp3). Furthermore, transforming growth factor (TGF)- $\beta 1$ promotes Treg differentiation, which in turn suppresses adaptive T-cell responses and prevents autoimmunity. An imbalance between Th17 and Tregs is characteristic for ARDS (7-9). Pharmacologically restoring the equilibrium between Th17 and Tregs has therefore become a promising therapeutic strategy for ARDS. Cyclic adenosine monophosphate (cAMP) signaling is a major pathway as well as a key therapeutic target for inducing immune tolerance and is involved in Treg function. Control of effector T-cell (Teff) proliferation and function by cAMP signaling is well established. Several mechanisms exist to suppress Teff cells by activation of cAMP signaling, including the action of anti-inflammatory agents through $\mathrm{G}$ protein-coupled receptors, cell-to-cell interactions and inhibition of cyclic nucleotide phosphodiesterases (PDEs) within cells. In addition, cAMP can also suppress TGF- $\beta$-mediated adaptive Treg differentiation and reduce the Treg concentration (10-12). It therefore appeared that targeting the cAMP signaling pathway to reverse the imbalance of Treg/Th17 may represent a promising approach for ARDS therapy. 
The present study showed that activation of cAMP partially restored the Treg/Th17 ratio in a mouse model of ARDS and prevented the progression of the condition. Targeting of the cAMP signaling pathway was therefore indicated to be a promising strategy for ARDS therapy.

\section{Materials and methods}

Experimental protocols and animals. The present study was approved by the ethics committee of Zhejiang University (Hangzhou, China). Male Kunming mice (age, 8 weeks; weight, 20-22 g) were purchased from the Animal Research Center of Zhejiang University (Hangzhou, China) and housed in the laboratory animal center of Zhejiang University at $22^{\circ} \mathrm{C}$ with $40-60 \%$ humidity under a 12 -h light/dark cycle. Mice were allowed access to standard food and clean water ad libitum. Mice were randomly divided into three groups $(n=10)$ : i) Sham group: Mice were administered with normal saline, then subjected to sham surgery; ii) ARDS group: Mice were supplemented with normal saline and then underwent cecal ligature and puncture (CLP); iii) ARDS+PTX group: Mice received PTX $10 \mathrm{mg} / \mathrm{kg}$ per hour for $5 \mathrm{~h}$ via intraperitoneal administration, followed by undergoing CLP. CLP was performed as follows: Subsequent to anesthesia with $8 \%$ sevoflurane (Shanghai Hengrui Pharmaceutical Co., Ltd., Shanghai, China), a midline laparotomy was performed. After the cecum was carefully isolated, bowel obstruction was prevented by placing a cotton ligature below the ileocecal valve. The cecum was then punctured twice with an 18-gauge needle. Animals in the Sham group were subjected to abdominal incision, while cecal ligation and perforation were not performed. Subsequently, the two layers of the abdominal cavity were closed with 3.0 silk sutures, followed by fluid resuscitation by subcutaneous administration of pre-warmed sterile saline ( $0.5 \mathrm{ml} / 10 \mathrm{~g}$ body weight). Animals in the Sham and CLP groups received tramadol (Shanghai Hengrui Pharmaceutical Co., Ltd.; $0.05 \mathrm{mg} / \mathrm{kg}$ body weight subcutaneously, repeated every $8 \mathrm{~h}$ ) for post-operative analgesia. The animals were then returned to their cages, where they received water and food. All of these procedures were performed by the same investigator to ensure consistency.

Twenty-four hours after lung injury, animals were sacrificed with $5 \mathrm{mg} / \mathrm{kg}$ tramadol; lungs and spleens were collected for further analysis. All animals used in the present study were housed and cared for in accordance with the Chinese Pharmacological Society Guidelines for Animal Use. All surgical procedures were performed under $8 \%$ sevoflurane anesthesia, and all efforts were made to minimize animal suffering.

Histological examination and hematoxylin and eosin $(H \& E)$ staining. The lung tissue was fixed in $4 \%$ paraformaldehyde (Beyotime Institute of Biotechnology, Haimen, China) and the pulmonary lobules were cut into 3 -mm thick blocks. Following embedding in paraffin, tissues were cut into $4-\mu \mathrm{m}$ slices. After de-paraffinization using xylene and re-hydration using a graded ethanol series, the sections were stained with H\&E (Bogoo, Shanghai, China) and images were captured under a microscope (BX41TF; Olympus Corporation, Tokyo, Japan). Tissue structure, inflammatory cell infiltration and necrosis were observed.
Isolation of spleen lymphocytes. The spleen was harvested and gently pressed through a $70-\mu \mathrm{m}$ cell strainer (BD Biosciences, Franklin Lakes, NJ, USA), followed by washing with $0.9 \%$ sodium chloride solution and red cell lysis with ACK Lysing Buffer (Beyotime Institute of Biotechnology). Among these splenocytes, $\mathrm{CD} 4^{+} \mathrm{CD} 25^{+} \mathrm{Foxp} 3^{+}$Tregs, $\mathrm{CD} 4^{+} \mathrm{IL}-17^{+}$Th17 cells and $\mathrm{CD} 4^{+}$cells were identified using fluorescence-assisted cell sorting (FACS; Attune NxT; Thermo Fisher Scientific, Inc., Waltham, MA, USA). cAMP was quantified using a cAMP-specific ELISA (Direct cAMP ELISA; cat. no. ADI900-066; Enzo Life Sciences, Farmingdale, NY, USA).

Western blot analysis. Tissue and lymphocyte lysates were prepared and western blotting was performed as previously described (13). In brief, the protein concentration was determined using BCA Protein assay kit (Beyotime Institute of Biotechnology) and $30 \mu \mathrm{g}$ protein in each lane was separated on $10 \%$ sodium dodecyl polyacrylamide gels and electrotransferred onto nitrocellulose membranes (EMD Millipore, Billerica, MA, USA). Membranes were then probed with rabbit polyclonal anti-Foxp3 (1:1,000; Abcam, Cambridge, MA, USA; cat. no. ab54501), rabbit polyclonal anti-RAR-related orphan receptor $\gamma \mathrm{t}$ (ROR $\gamma \mathrm{t}$; 1:1,000; Abcam; cat. no. 78007) or rabbit monoclonal anti-signal transducer and activator of transcription (STAT)3 (1:1,000; Cell Signaling Technologies, Inc., Danvers, MA, USA; cat. no. 12640) as well as anti-glyceraldehyde-3-phosphate dehydrogenase (GAPDH) antibodies (1:1,000; Santa Cruz Biotechnology, Inc., Dallas, TX, USA; cat. no. sc-25778) for $12 \mathrm{~h}$ at $4^{\circ} \mathrm{C}$. Subsequently, membranes were incubated with horseradish peroxidase-conjugated mouse anti-rabbit secondary antibody (1:2,000; cat. no. sc-2357; Santa Cruz Biotechnology, Inc.) for $1 \mathrm{~h}$ at $37^{\circ} \mathrm{C}$, and SuperSignal West Pico Chemiluminescent Substrate (Pierce Biotechnology, Inc., Rockford, IL, USA) according to the manufacturer's instructions.

Reverse-transcription quantitative polymerase chain reaction $(R T-q P C R)$. Total RNA was extracted with TRIzol according to the manufacturer's instructions and was reverse-transcribed using the Prime Script ${ }^{\mathrm{TM}}$ RT reagent kit (Takara Bio, Inc., Otsu, Japan). The $10 \mu 1$ RT mixture contained $400 \mathrm{ng}$ of total RNA, $2 \mu 1$ 5X PrimeScript Buffer, $0.5 \mu$ l PrimeScript RT Enzyme mix I; $0.5 \mu \mathrm{l}$ Oligo dT Primer $(50 \mu \mathrm{M}), 0.5 \mu 1$ random hexamers $(100 \mu \mathrm{M})$ and RNase free distilled water to $10 \mu \mathrm{l}$. The complementary DNA template was amplified by real-time PCR using the SYBR-PremixExTaq ${ }^{\mathrm{TM}}$ kit (Takara Bio, Inc.) on a StepOne Real-Time PCR System (Applied Biosystems; Thermo Fisher Scientific, Inc.). The PCR reaction mixture contained $12.5 \mu 1$ 2X SYBR Premix Ex Taq, $0.5 \mu 1$ forward primer $(10 \mu \mathrm{M}), 0.5 \mu \mathrm{l}$ reverse primer $(10 \mu \mathrm{M}), 2 \mu \mathrm{l}$ cDNA and $9.5 \mu \mathrm{l}$ distilled water. The primers were obtained from Sangon Biotech Co., Ltd. (Shanghai, China) and the sequences were as follows: 5'-GTGGCCCGGATGTGAGAAG-3 (forward), 5'-GGAGCCCTTG TCGGATGATG-3 (reverse) for Foxp3; 5'-TCAAGTTTGGCCGAATGTC-3' (forward), 5'-CATCTG AGAGCCCTAAAGTG TATG-3' (reverse) for ROR $\gamma \mathrm{t}$; 5'-GCA CCGTCAAGGCTG AGAAC-3' (forward), 5'-GCCTTCTCC ATGGTGGTGAA-3' (reverse) for GAPDH. Thermal cycling was programmed as follows: $95^{\circ} \mathrm{C}$ for $30 \mathrm{sec}$ followed by 40 
A

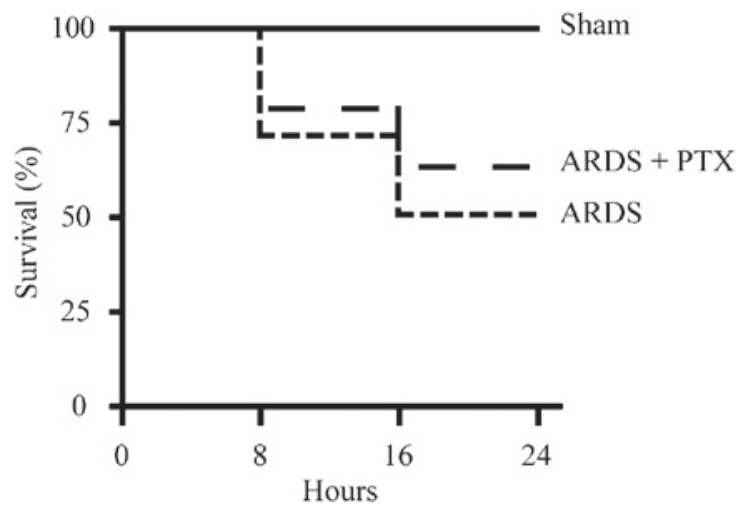

C

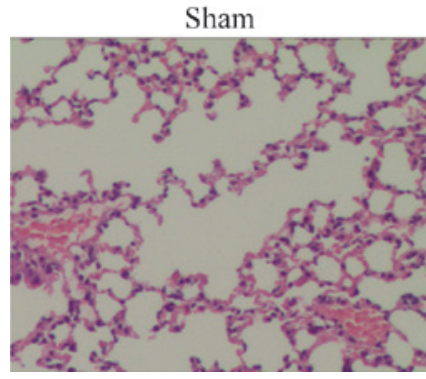

ARDS
B
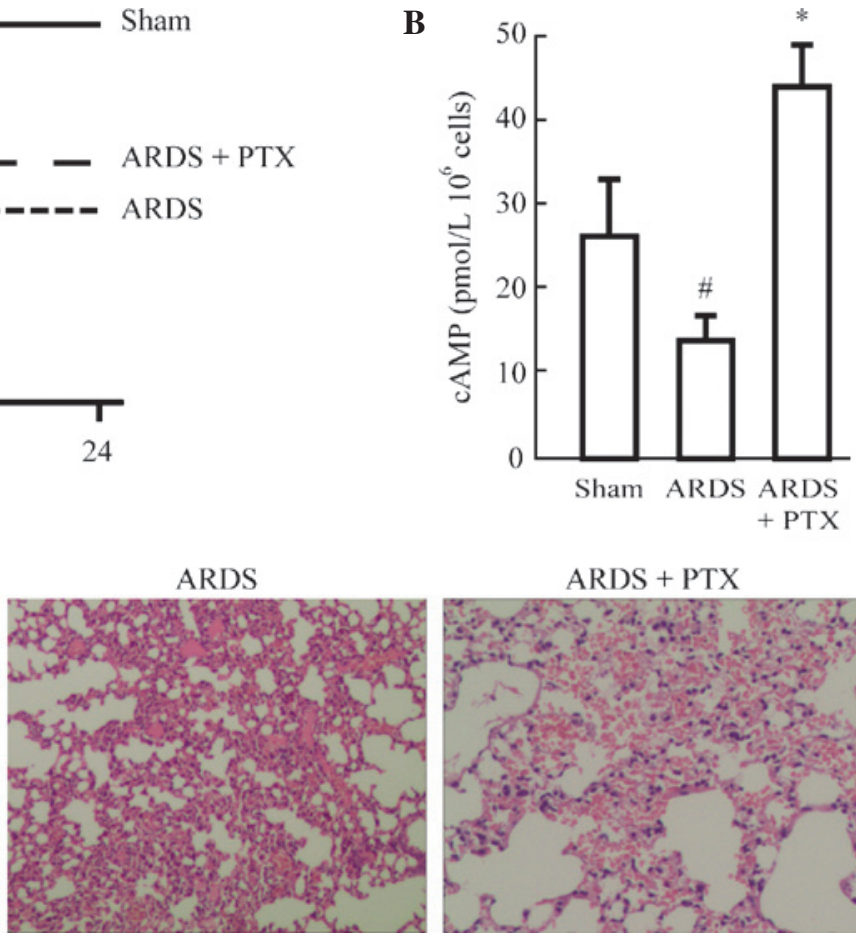

ARDS + PTX

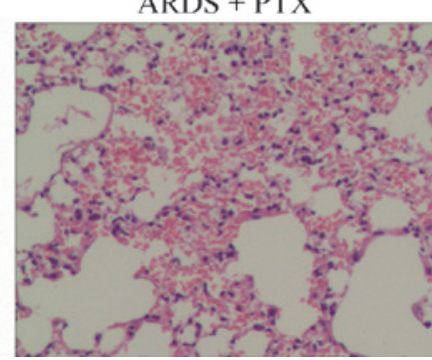

Figure 1. Activation of cAMP signaling attenuates CLP-induced acute lung injury. (A) Pre-treatment with phosphodiesterase antagonist PTX improved the survival rate in a mouse model of ARDS induced by CLP. The percentage of surviving animals at $24 \mathrm{~h}$ after surgery is shown. (B) The cAMP concentration in the spleen lymphocytes of mice from different treatment groups, from lysates of $10^{6}$ cells in each group. (C) Hepatic morphological changes were examined by hematoxylin and eosin staining. Representative images are shown for each group (original magnification, x100). CLP induced alveolar septal thickening, accumulation of inflammatory cells in the interstitium and alveoli and an influx of protein-rich fluid into the alveolar space, which was obviously attenuated by PTX. Values are expressed as the mean \pm standard deviation ( $\mathrm{n}=5$ at least in each group). ${ }^{*} \mathrm{P}<0.01 \mathrm{vs.} \mathrm{Sham} \mathrm{group;}{ }^{*} \mathrm{P}<0.05$ vs. ARDS group. cAMP, cyclic adenosine monophosphate; PTX, pentoxifyllinum; CLP, cecal ligature and puncture; ARDS, acute respiratory distress syndrome.

cycles of $95^{\circ} \mathrm{C}$ for $5 \mathrm{sec}, 60^{\circ} \mathrm{C}$ for $20 \mathrm{sec}$ and $72^{\circ} \mathrm{C}$ for $15 \mathrm{sec}$, and subsequent final elongation at $72^{\circ} \mathrm{C}$ for $10 \mathrm{~min}$. Gene expression was assessed using the $2^{-\Delta \Delta \mathrm{Cq}}$ method (14) and levels of the analyte mRNAs were normalized to those of GAPDH as an internal standard.

Cytokine analysis. IL-2, IL-6, IL-10, IL-17 and TGF- $\beta$ secretion by spleen lymphocytes was quantified using an Inflammation Kit (BD Biosciences) according to the manufacturer's recommendations.

Statistical analysis. Values are expressed as the mean \pm standard deviation. Statistical analyses were conducted on Prism 6.0 (GraphPad Software, Inc., La Jolla, CA, USA) using one-way analysis of variance or the unpaired Student's $t$-test as indicated. $\mathrm{P}<0.05$ was considered to indicate a statistically significant difference.

\section{Results}

Activation of cAMP signaling attenuates CLP-induced ALI. In order to identify whether activation of the cAMP signaling pathway can ameliorate ALI and the resultant ARDS, one group of animals was pre-treated with PDE inhibitor pentoxifylline (PTX). As show in Fig. 1A, although the difference was not significant, PTX pre-treatment improved the survival rate of mice following CLP to a certain degree (from 50 to $61 \%$ ). In addition, cAMP levels were significantly reduced in the splenocytes of mice following CLP, while they were significantly increased in splenocytes from animals pre-treated with PTX compared with those in the Sham group (Fig. 1B). Furthermore, the degree of lung injury was evaluated in mice treated with or without PTX prior to CLP. Histological analysis revealed that CLP induced alveolar septal thickening, accumulation of inflammatory cells in the interstitium and alveoli and an influx of protein-rich fluid into the alveolar space. However, mice pre-treated with PTX demonstrated reduced structural changes following CLP (Fig. 1C).

PTX partly restores the Treg/Th17 imbalance in mice with $C L P$-induced ARDS. FACS analysis revealed that the proportion of $\mathrm{CD} 4^{+} \mathrm{CD} 25^{+} \mathrm{Foxp}^{+}$Tregs and CD $4^{+} \mathrm{IL}-17^{+}$Th17 cells in spleen lymphocytes were significantly increased after CLP (Fig. 2), which was consistent with the results of previous studies $(15,16)$. However, in CLP-induced mouse models of ARDS, the Treg/Th17 ratio was obviously decreased compared with that in the Sham group, which suggested that the Treg/Th17 balance was shifted towards Th17 (Fig. 3). Of note, pre-treatment with PTX significantly attenuated CLP-induced increases in the number of Tregs and Th17 as well as the decrease of the Treg/Th17 ratio.

Activation of cAMP signaling decreases CLP-induced cytokine secretion. Inflammation is the main pathological consequence of CLP-induced sepsis, which is characterized by the release of inflammatory factors and contributes to ARDS. Thus, the ability of PTX to prevent cytokine production of spleen lymphocytes following CLP was assessed. An ELISA 

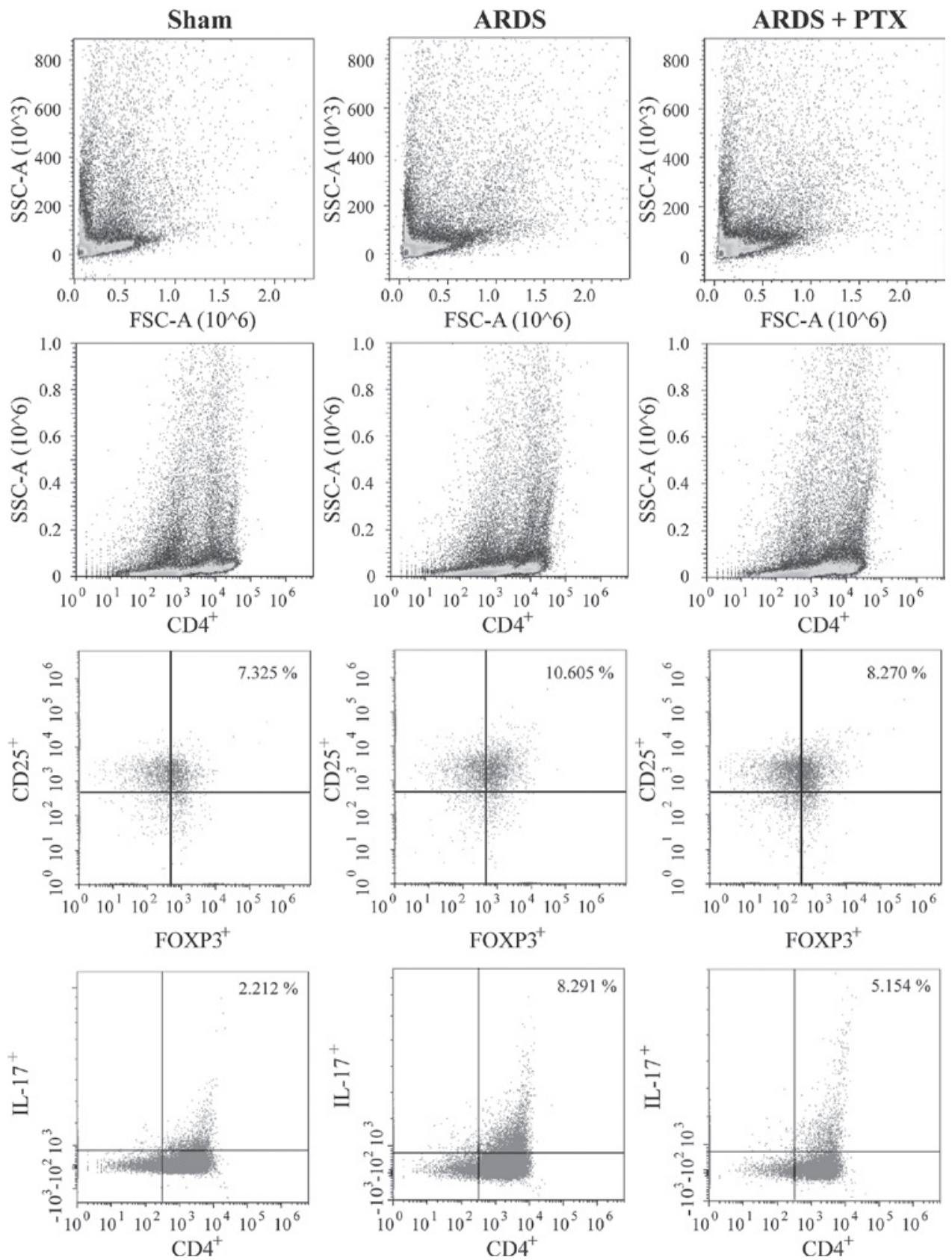

Figure 2. PTX treatment attenuates cecal ligature and puncture-induced increases in the number of Tregs and Th17 cells. Analysis of differentiated $\mathrm{CD} 4^{+} \mathrm{CD} 25^{+} \mathrm{Foxp} 3^{+}$Tregs and CD4 ${ }^{+} \mathrm{IL}-17^{+} \mathrm{Th} 17$ cells among spleen lymphocytes by fluorescence-assisted cell sorting. Representative scatter plots are shown. PTX, pentoxifyllinum; Treg, T-regulatory cell; IL-17, interleukin 17; Th17, IL-17-producing T helper lymphocyte; FOXP3, forkhead box P3; ARDS, acute respiratory distress syndrome; SSC, side scatter; FSC, forward scatter.

revealed that the secretion of the classic inflammatory factors IL-2, IL-6, IL-10, IL-17 and TGF- $\beta$ was significantly upregulated following CLP. However, PTX treatment significantly attenuated CLP-induced upregulation of IL-2, IL-6, IL-10, IL-17 $(\mathrm{P}<0.01)$. While PTX also decreased TGF- $\beta$, levels compared to those in the ARDS group, differences were not significant (Fig. 4).

PTX attenuates CLP-induced expression of Foxp 3 and ROR $\gamma t$ as well as activation of STAT3. To investigate the underlying mechanisms of the PTX-induced reduction of cytokine production and partial restoration of the Treg/Th17 balance following CLP, Foxp3, ROR $\gamma$ t and STAT3 were assessed as members of the cAMP signaling pathway. As shown in the Fig. 5, Foxp3 and
ROR $\gamma \mathrm{t}$ expression were significantly increased at the protein and mRNA level following CLP; furthermore, the protein levels of activated STAT3 were obviously increased. However, pre-treatment with PTX obviously attenuated these increases. These results may indicate that the observed PTX-induced decreases in cytokine levels and restoration of the Treg/Th17 balance following CLP may be due to decreasing Foxp3 and ROR $\gamma \mathrm{t}$ expression as well as the levels of activated STAT3, which is consistent with the findings of a previous study (17).

\section{Discussion}

The present study provided a novel strategy for the treatment of ARDS by specifically interfering with cAMP-associated 


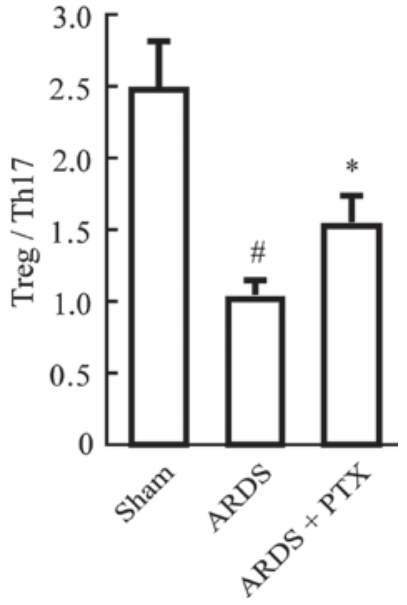

Figure 3. PTX treatment restores the ratio of Treg/Th17 in mice with ARDS Values are expressed as the mean \pm standard deviation $(n=5$ at least in each group). " $\mathrm{P}<0.01$ vs. Sham group; ${ }^{*} \mathrm{P}<0.05$ vs. ARDS group. PTX, pentoxifyllinum; Treg, T-regulatory cell; Th17, interleukin 17-producing T helper lymphocyte; FOXP3, forkhead box P3; ARDS, acute respiratory distress syndrome.

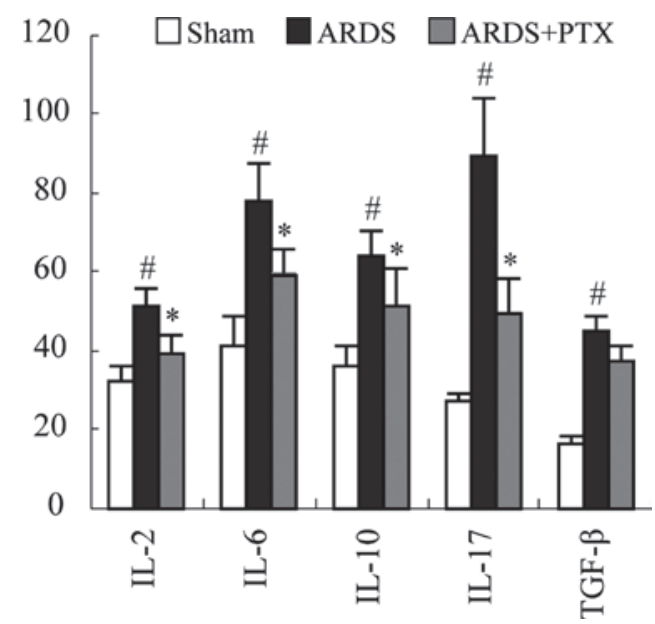

Figure 4. PTX reduces the secretion of IL-2, IL-6, IL-10, IL-17 and TGF- $\beta$ in mice with ARDS. The supernatant was collected from the culture of spleen lymphocytes isolated from mice from the different treatment groups and the cytokine levels were assessed using an ELISA kit. Values are expressed as the mean \pm standard deviation ( $\mathrm{n}=5$ at least in each group). ${ }^{"} \mathrm{P}<0.01 \mathrm{vs}$. sham group; ${ }^{*} \mathrm{P}<0.05$ vs. ARDS group. PTX, pentoxifyllinum; ARDS, acute respiratory distress syndrome; IL, interleukin; TGF, transforming growth factor.

signaling. It was revealed that pre-treatment with the PDE antagonist PTX obviously attenuated lung injury and mortality, and reduced the production of IL-2, IL-6 IL-10, IL-17 and TGF- $\beta$ in a mouse model of CLP-induced ARDS. To investigate the underlying mechanism, the lymphocytes from the spleens of the mice were isolated and analyzed, revealing that the number of Tregs as well as that of Th17 cells was increased after CLP, while the Treg/Th17 ratio was shifted towards Th17. Of note, pre-treatment with PTX decreased the number of Tregs and Th17 cells and partially restored the Treg/Th17 ratio. Furthermore, while cAMP levels were significantly decreased in the splenocytes of mice following CLP, pre-treatment with PTX led to a marked increase of cAMP. Furthermore, PTX decreased CLP-induced increases in Foxp3 and ROR $\gamma$ t expression as well as STAT3 activation.
A

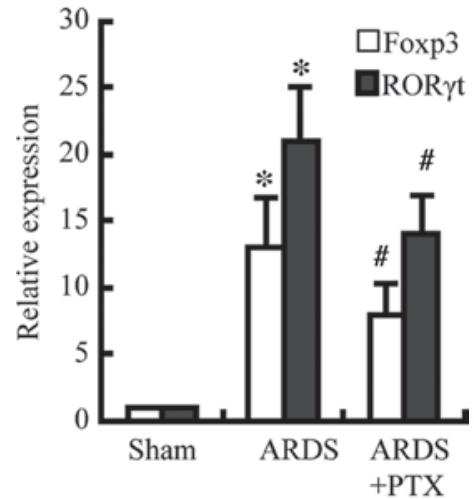

B

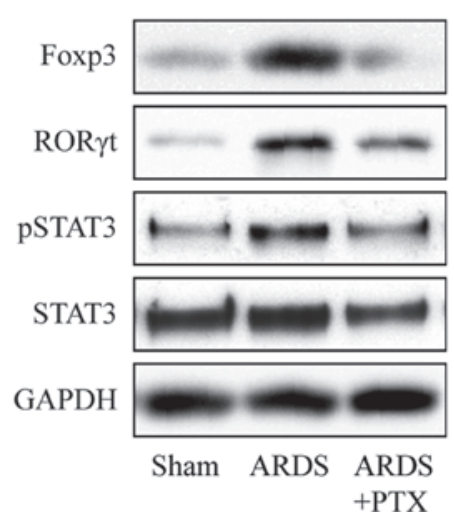

Figure 5. Effects of PTX on the expression of Foxp3 and ROR $\gamma$ t. (A) Foxp3 and ROR $\gamma \mathrm{t}$ mRNA levels of spleen lymphocytes isolated from mice from the different treatment groups were determined by polymerase chain reaction analysis with normalization to GAPDH. Values are expressed as the mean \pm standard deviation. (B) Foxp3, ROR $\gamma \mathrm{t}$, pSTAT3 and STAT3 protein levels were detected by western blot analysis with GAPDH used as a loading control. The experiment was repeated three times. " $\mathrm{P}<0.01$ vs. the sham group; ${ }^{\text {P }}<0.01$ vs. the ARDS group.pSTAT3, phosphorylated signal transducer and activator of transcription; FOXP3, forkhead box P3; ARDS, acute respiratory distress syndrome; PTX, pentoxifyllinum; GAPDH, glyceraldehyde-3-phosphate dehydrogenase; ROR $\gamma \mathrm{t}$, RAR-related orphan receptor $\gamma \mathrm{t}$.

ARDS is a fatal but potentially reversible clinical syndrome of lung inflammation caused by numerous direct and indirect lung insults. Pulmonary and extra-pulmonary infection, aspiration and trauma are common causes of ARDS. ARDS is also increasingly recognized as a Th17- and Treg-associated immune disease. The present study found that the proportion of $\mathrm{CD} 4^{+} \mathrm{CD} 25^{+} \mathrm{Foxp} 3^{+}$Tregs amongst all $\mathrm{CD}^{+}$splenocytes increased in mice with ARDS induced by CLP, which was consistent with the results of previous studies. Adamzik et al (15) reported that the ratio of Tregs amongst all $\mathrm{CD} 4^{+}$lymphocytes in patients succumbed to ARDS (16.5\%; $\mathrm{P}=0.025)$ was almost two-fold greater than that in ARDS survivors $(9.0 \%$; $\mathrm{P}=0.015)$ compared to that in control subjects (5.9\%). Furthermore, D'Alessio et al (16) showed that the number of $\mathrm{CD} 4{ }^{+} \mathrm{CD} 25^{+} \mathrm{Foxp} 3^{+}$Tregs was increased in mice with lipopolysaccharide-induced lung injury and remained elevated until day 10 . Of note, administration of $\mathrm{CD} 4{ }^{+} \mathrm{CD} 25^{+} \mathrm{Foxp}^{+}$Tregs was able to remedy experimental lung injury (16). These observations may indicate that $\mathrm{CD}^{+}{ }^{+} \mathrm{CD} 25^{+} \mathrm{Foxp}^{+}$Tregs are a double-edged sword in ARDS: While a modest increase of Tregs may have anti-inflammatory action, excessive increases of Tregs may lead to tissue injury. While the roles of Th17 cells in ARDS have remained largely 
elusive, they have been investigated in several types of lung disease (18-20). Recently, Yu et al (21) showed that ARDS patients exhibited a significant increase in the frequency of Th17 cells and their signature cytokine, IL-17. The present study revealed that PTX decreased the percentage of Tregs and Th17 cells in spleen lymphocytes, and partially restored the Treg/Th17 ratio. The transcriptional regulators Foxp3 and ROR $\gamma \mathrm{t}$ are important for regulating the differentiation and function of Treg/Th17 (22,23), and the present study found that PTX significantly inhibited the expression of Foxp3 and ROR $\gamma \mathrm{t}$.

In conclusion, the present study provided a novel treatment strategy for ARDS by restoring the Treg/Th17 balance and the subsequent immune response via cAMP signaling, which requires pre-clinical and clinical validation.

\section{Acknowledgements}

The present study was supported by Natural Science Foundation of Zhejiang Province (grant no. LQ12H01002) and the Zhejiang Provincial Medical and Health Platform Key Program (grant no. 2012ZDA002).

\section{References}

1. Ashbaugh DG, Bigelow DB, Petty TL and Levine BE: Acute respiratory distress in adults. Lancet 2: 319-323, 1967.

2. ARDS Definition Task Force, Ranieri VM, Rubenfeld GD, Thompson BT, Ferguson ND, Caldwell E, Fan E, Camporota L and Slutsky AS: Acute respiratory distress syndrome: The Berlin Definition. JAMA 307: 2526-2533, 2012.

3. Pierrakos C, Karanikolas M, Scolletta S, Karamouzos V and Velissaris D: Acute respiratory distress syndrome: Pathophysiology and therapeutic options. J Clin Med Res 4: 7-16, 2012.

4. Curley GF and Laffey JG: Future therapies for ARDS. Intensive Care Med 41: 322-326, 2015.

5. Besnard AG, Togbe D, Couillin I, Tan Z, Zheng SG, Erard F, Le Bert M, Quesniaux V and Ryffel B: Inflammasome-IL-1-Th17 response in allergic lung inflammation. J Mol Cell Biol 4: 3-10, 2012.

6. Tsai HC, Velichko S, Hung LY and Wu R: IL-17A and Th17 cells in lung inflammation: An update on the role of Th17 cell differentiation and IL-17R signaling in host defense against infection. Clin Dev Immunol 2013: 267971, 2013.

7. Ji L, Zhan Y, Hua F, Li F, Zou S, Wang W, Song D, Min Z, Chen $\mathrm{H}$ and Cheng Y: The ratio of Treg/Th17 cells correlates with the disease activity of primary immune thrombocytopenia. PLoS One 7: e50909, 2012.
8. Kimura A and Kishimoto T: IL-6: Regulator of Treg/Th17 balance. Eur J Immunol 40: 1830-1835, 2010.

9. Ma L, Xue HB, Guan XH, et al: The Imbalance of Th17 cells and CD4(+) CD25(high) Foxp3(+) Treg cells in patients with atopic dermatitis. J Eur Acad Dermatol Venereol 28: 1079-1086, 2014.

10. Bourne HR, Lichtenstein LM, Melmon KL, Henney CS, Weinstein Y and Shearer GM: Modulation of inflammation and immunity by cyclic AMP. Science 184: 19-28, 1974.

11. Cao J, Zhang X, Wang Q, Wang X, Jin J, Zhu T, Zhang D, Wang W, Li X, Li Y, et al: Cyclic AMP suppresses TGF- $\beta$-mediated adaptive Tregs differentiation through inhibiting the activation of ERK and JNK. Cell Immunol 285: 42-48, 2013.

12. Lee J, Kim TH, Murray F, et al: Cyclic AMP concentrations in dendritic cells induce and regulate Th2 immunity and allergic asthma. Proc Natl Acad Sci USA 112: 1529-1534, 2015.

13. Ding XF, Zhou J, Hu QY, Liu SC and Chen G: The tumor suppressor pVHL down-regulates never-in-mitosis A-related kinase 8 via hypoxia-inducible factors to maintain cilia in human renal cancer cells. J Biol Chem 290: 1389-1394, 2015.

14. Livak KJ and Schmittgen TD: Analysis of relative gene expression data using real-time quantitative PCR and the 2(-Delta Delta C(T)) method. Methods 25: 402-408, 2001.

15. Adamzik M, Broll J, Steinmann J, Westendorf AM, Rehfeld I, Kreissig C and Peters J: An increased alveolar CD4+CD25+Foxp3+T-regulatory cell ratio in acute respiratory distress syndrome is associated with increased 30-day mortality. Intensive Care Med 39: 1743-1751, 2013.

16. D'Alessio FR, Tsushima K, Aggarwal NR, West EE, Willett MH, Britos MF, Pipeling MR, Brower RG, Tuder RM, McDyer JF and King LS: CD4+CD25+Foxp3+ Tregs resolve experimental lung injury in mice and are present in humans with acute lung injury. J Clin Invest 119: 2898-2913, 2009.

17. Zhang F, Fuss IJ, Yang Z and Strober W: Transcription of ROR $\gamma t$ in developing Th17 cells is regulated by E-proteins. Mucosal Immunol 7: 521-532, 2014.

18. Newcomb DC and Peebles RS Jr: Th17-mediated inflammation in asthma. Curr Opin Immunol 25: 755-760, 2013.

19. Kononova TE, Urazova OI, Novitskii VV, Churina EG, Kolobovnikova YV, Ignatov MV, Zakharova PA and Pechenova OV: Functional activity of Th-17 lymphocytes in pulmonary tuberculosis. Bull Exp Biol Med 156: 743-745, 2014.

20. Vargas-Rojas MI, Ramírez-Venegas A, Limón-Camacho L, Ochoa L, Hernández-Zenteno R and Sansores RH: Increase of Th17 cells in peripheral blood of patients with chronic obstructive pulmonary disease. Respir Med 105: 1648-1654, 2011.

21. Yu ZX, Ji MS, Yan J, Cai Y, Liu J, Yang HF, Li Y, Jin ZC, Zheng JX: The ratio of Th17/Treg cells as a risk indicator in early acute respiratory distress syndrome. Crit Care 19: 82, 2015.

22. Chen Z, Lin F, Gao Y, Li Z, Zhang J, Xing Y, Deng Z, Yao Z, Tsun A and Li B: FOXP3 and ROR $\gamma \mathrm{t}$ : Transcriptional regulation of Treg and Th17. Int Immunopharmacol 11: 536-542, 2011.

23. Chu S, Zhong X, Zhang J, Lao Q, He Z and Bai J: The expression of Foxp3 and ROR gamma $t$ in lung tissues from normal smokers and chronic obstructive pulmonary disease patients. Int Immunopharmacol 11: 1780-1788, 2011. 